\title{
Ubisol Coenzyme Q10 promotes mitochondrial biogenesis in HT22 cells challenged by glutamate
}

\author{
MARY A. ZIMMERMAN ${ }^{1,2}$, MIA HALL ${ }^{1}$, QI QI ${ }^{1,3}$, SURESH L. MEHTA ${ }^{1,4}$, GUISHENG CHEN ${ }^{3}$ and P. ANDY LI ${ }^{1}$ \\ ${ }^{1}$ Department of Pharmaceutical Sciences, Biomanufacturing Research Institute Biotechnology Enterprise (BRITE), \\ North Carolina Central University, Durham, NC 27707; ${ }^{2}$ Department of Biology, University of Wisconsin La Crosse, \\ La Crosse, WI 54601, USA; ${ }^{3}$ Department of Neurology, General Hospital of Ningxia Medical University, \\ Ningxia Key Laboratory of Cerebrocranial Diseases, Incubation Base of National Key Laboratory, \\ Yinchuan, Ningxia 750004, P.R. China; ${ }^{4}$ Department of Neurological Surgery, \\ University of Wisconsin School of Medicine and Public Health, Madison, WI 53792, USA
}

Received March 20, 2020; Accepted July 15, 2021

DOI: 10.3892/etm.2021.10730

\begin{abstract}
Glutamate-induced excitotoxicity is a wellrecognized cause of neuronal cell death. Nutritional supplementation with Coenzyme Q10 (CoQ10) has been previously demonstrated to serve neuro-protective effects against glutamate-induced excitotoxicity. The aim of the present study was to determine whether the protective effect of CoQ10 against glutamate toxicity could be attributed to stimulating mitochondrial biogenesis. Mouse hippocampal neuronal HT22 cells were incubated with glutamate with or without ubisol Q10. The results revealed that glutamate significantly decreased levels of mitochondrial biogenesis related proteins, including peroxisome proliferator-activated receptor gamma coactivator (PGC)-1 $\alpha$ and nuclear respiratory factor (NRF)2. Additionally, glutamate reduced mitochondrial biogenesis, as determined using a mitochondrial biogenesis kit. Pretreatment with CoQ10 prevented decreases in phosphorylated (p)-Akt, p-cAMP response element-binding protein, PGC-1 $\alpha$, NRF2 and mitochondrial transcription factor A, increasing
\end{abstract}

Correspondence to: Professor P. Andy Li, Department of Pharmaceutical Sciences, Biomanufacturing Research Institute Biotechnology Enterprise (BRITE), North Carolina Central University, Building 2031, 1801 Fayetteville Street, Durham, NC 27707, USA

E-mail: pli@nccu.edu

Abbreviations: CNS, central nervous system; CREB, cAMP response element-binding protein; CoQ10, coenzyme Q10; COX-I, cyclooxygenase I; ETC, electron transport chain; mPTP, mitochondrial permeability transition pore; NAD, nicotinamide adenine dinucleotide; NRF, nuclear respiratory factor; PGC-1 $\alpha$, peroxisome proliferator-activated receptor gamma coactivator- $1 \alpha$; ROS, reactive oxygen species; SDH-A, succinate dehydrogenase complex flavoprotein subunit A; TFAM, mitochondrial transcription factor A

Key words: coenzyme Q10, excitotoxicity, glutamate, mitochondrial biogenesis mitochondrial biogenesis. Taken together, the results described a novel mechanism of CoQ10-induced neuroprotection and indicated a central role for mitochondrial biogenesis in protecting against glutamate-induced excitotoxicity.

\section{Introduction}

Glutamate excitotoxicity is a condition in which excessive glutamate accumulates in the central nervous system (CNS) causing acute neuronal injury and long-term neurodegeneration (1). Abrupt increases in glutamate are known to accompany traumatic brain injuries and cerebral ischemia, as well as to contribute to neurodegenerative diseases, such as amyotrophic lateral sclerosis, fibromyalgia, multiple sclerosis, Alzheimer's, Parkinson's, and Huntington's diseases. Glutamate-induced injury is primarily the result of increased intracellular calcium levels facilitated by the engagement of glutamate receptors (2). In addition to $\mathrm{Ca}^{2+}$ overload, receptor stimulation also leads to collapse of the electrochemical gradient, and activation of protein kinases and endonucleases. These alterations accelerate cell death through multiple pathways and through degradation of important substances (3). Work in our own lab has shown that HT22 mouse hippocampal cells exposed to glutamate experience mitochondrial dysfunction causing formation of the mitochondrial permeability transition pore (mPTP). This occurred with increased calcium retention, alteration of the mitochondrial membrane potential (MMP), fragmentation of the mitochondria, release of apoptosis inducing factor (AIF), and breakdown of DNA $(4,5)$.

Much work has been done on how to best limit the effects of glutamate to prevent overstimulation of its receptors. We have found that supplementation with coenzyme Q10 (CoQ10) can prevent many of the glutamate- or rotenone-induced changes within the mitochondria, and improve viability of neurons (4,6-9).

One of the principal defenses against mitochondrial dysfunction is the detoxification of reactive oxygen species (ROS) whose stimulation is both triggered and enhanced when the mitochondrial membrane potential is disrupted. CoQ10 is a 
major cofactor of the electron transport chain (ETC) where majority ROS are produced. Within the ETC, CoQ10 plays a key role in transporting electrons between complexes I, II and III. It is also recognized as having antioxidant functions. Thus, when ETC function is disrupted by a mitochondrial insult, CoQ10's location positions itself as a key antioxidant to reduce oxidative damage (10). However, continuous, high levels of oxidative stress can lead to CoQ10 depletion and prevent adequate detoxification of ROS.

Therefore, exogenous antioxidant supplementation of CoQ10, can improve outcomes after mitochondrial damage (6-8), including damage caused by glutamate (4). CoQ10 is produced naturally within cells as part of the cholesterol pathway, but the cellular content can be further increased through consumption of food sources high in CoQ10, such as meat, nuts and green leafy vegetables. Exogenous ubiquinone (oxidized form of CoQ10), or ubiquinol [a reduced and more bioavailable form of CoQ10 (11)], can also be administered. This additional supplementation may particularly benefit aging adults as CoQ10 levels naturally decline with age (12).

The benefits from CoQ10 supplementation have been documented in a number of clinical trials. For example, in chronic heart failure patients, CoQ10 decreased mortality, reduced the incidence of hospitalization, and improved patients' functional classification by the New York Heart Association (13). CoQ10 increases total capacity of antioxidant enzymes and reduces inflammatory biomarkers in diabetic hemodialysis patients $(14,15)$. CoQ10 benefits have also been reported in a clinical trial of multiple sclerosis (16). More clinical trials are needed to establish the efficacy of CoQ10 in preventing neurodegeneration and in preserving mitochondrial function. Our lab has been interested in the neuroprotective benefits of CoQ10 supplementation. We believe this protection can be attributed to the effects of CoQ10 on the mitochondria, beyond antioxidant functions. In this study, our goal was to determine whether CoQ10 could increase mitochondrial biogenesis to improve the outcome against glutamate toxicity.

Mitochondrial biogenesis is a process by which new mitochondria are formed through the growth and division of pre-existing mitochondria (17). Activation of this process often occurs during an insult to the cell. This is an attempt to counteract the damage process. Essentially, increasing numbers of mitochondria serves to boost ATP production and to increase detoxification of ROS. Unfortunately, this response is usually not sufficient to counteract the damage process (18). However, because of its protective effect, enhancing the mitochondrial biogenesis could be used as a strategy to protect cells against various damages including neurological diseases (19).

Changes in mitochondrial biogenesis can be determined by examining expression of key proteins mediating mitochondrial biogenesis pathways. Peroxisome proliferator-activated receptor gamma coactivator- $1 \alpha(\mathrm{PGC}-1 \alpha)$ appears to be the master regulator of mitochondrial biogenesis based on current knowledge (17). Once being activated, either through phosphorylation or deacetylation, PGC- $1 \alpha$ activates the mitochondrial transcription factors, nuclear respiratory factor 1 and 2 (NRF1,2). The NRF 1,2 could activate the mitochondrial NDA and protein synthesis either directly or through activating mitochondrial transcription factor A (TFAM) (20). Activation of these transcription factors by PGC-1 $1 \alpha$, leads to the synthesis of mitochondrial genes encoded in nuclear and mitochondrial DNA, promoting generation of new mitochondria.

The overall objectives of this work were to determine whether glutamate exposure itself affected mitochondrial biogenesis in hippocampal cells and whether CoQ10 supplementation conferred protection against glutamate-induced toxicity by promoting mitochondrial biogenesis. To this end, we measured mitochondrial biogenesis in hippocampal cell exposed to glutamate, with and without CoQ10 pre-treatment. We further analyzed mitochondrial biogenesis protein expression patterns to determine the molecular mechanisms involved. Given the active role mitochondria play in many disease models, including neuronal injury from glutamate excitotoxicity, we believe elucidating mitochondrial changes, and targeting them for correction, may improve therapeutic outcomes in patients.

\section{Materials and methods}

Cell culture. HT22 is a mouse hippocampal cell line kindly provided by Dr Panee at the University of Hawaii. HT22 cells were cultured as previously described (21). Stock preparations of glutamate (Sigma-Aldrich; Merck KGaA) and CoQ10 supplement, Ubisol-Q10 (Zymes LLC), dissolved in water, were diluted with cell culture media before being added to the cells. Glutamate concentrations (1-8 $\mathrm{mM}$ ) were tested in pilot study and $4 \mathrm{mM}$ was selected for subsequent studies because this concentration resulted in a $30 \%$ reduction of cell viability assessed by resazurin assay after $24 \mathrm{~h}$ exposure. Treatment of Ubisol-Q10 $(25 \mu \mathrm{g} / \mu \mathrm{l})$ was initiated $24 \mathrm{~h}$ prior to glutamate addition.

Reproductive potential assay. The reproductive potential of HT22 cells after exposure to glutamate and/or CoQ10 treatment was assessed using colony formation assay. Cells were treated as described above for untreated, glutamate, CoQ10, and glutamate plus CoQ10 experimental groups. After treatment, the cells were harvested and seeded at a density of 100 cells per $10 \mathrm{~cm}$ dish, except in the case of the glutamate only group, which were seeded at a higher density of 5,000 cells per dish. The cells were incubated under standard culture conditions for 14 days to allow visible colonies to form from individual cells seeded. The colonies were stained with a $0.05 \%$ crystal violet solution. Numbers of colonies were counted using ImageJ software version 1.49 (22). Plating efficiencies were calculated by taking the actual numbers of colonies formed in each plate and dividing by the number of cells originally seeded and then multiplying by 100 to obtain a percent. The percent of cells surviving was calculated by taking the individual plates' plating efficiencies and dividing by the average plating efficiency of the untreated, control group and again multiplying by 100 . The average percent survival for each group was quantified and analyzed for statistical significance using one-way ANOVA and Bonferroni's multiple comparisons test.

Western blotting. After undergoing experimental treatments for $24 \mathrm{~h}$ [untreated, glutamate alone $(4 \mathrm{mM}), \mathrm{CoQ} 10$ alone $(25 \mu \mathrm{g} / \mu \mathrm{l})$, and glutamate plus CoQ10 $24 \mathrm{~h}$ pre-treatment], cells were harvested and washed with phosphate-buffered 
saline (PBS) before lysing to obtain either total protein extracts, or protein fractions from cytosolic, mitochondrial, and nuclear compartments as previously described $(21,23)$. Protein concentrations were measured using standard Bradford assays (Bio-Rad Laboratories). Western blot analysis was performed essentially as previously described (21). Equal amount of protein $(20 \mu \mathrm{g})$ samples were loaded and separated on 4-12\% Bis-Tris NuPAGE gels (Invitrogen; Thermo Fisher Scientific, Inc.) and then transferred to Polyvinylidene fluoride membranes. The following primary antibodies from Cell Signaling Technology were used at 1:1,000 dilutions: Anti-AKT, anti-p-AKT, anti-CREB, anti-p-CREB, anti-COX-IV, and anti-Histone 3. Anti-PGC-1 $\alpha(1: 1,000)$ was obtained from Abcam. Anti-NRF2 (1:200) was from Santa Cruz Biotechnology and anti-TFAM $(1: 1,000)$ was from Calbiochem. Both anti-Histone 3 and $\beta$-actin $(1: 5,000)$ were used as protein loading controls. IRDye 680LT goat anti-rabbit, IRDye $800 \mathrm{CW}$ goat anti-mouse, and donkey anti-goat secondary antibodies from Li-COR, Inc. were used at 1:10,000 dilutions for visualization using the Li-COR Odyssey Classic Imaging System scanner. Protein bands were analyzed with the Li-COR Image Studio Software version 5.2.5. as previously described (21). Statistical significance was measured using one-way ANOVA and Bonferroni's multiple comparisons test.

Measurement of mitochondrial biogenesis. Different levels of mitochondrial biogenesis among experimental groups were assessed using a MitoBiogenesis ${ }^{\mathrm{TM}}$ In-Cell ELISA Kit (Abcam) according to the manufacturer's protocol. Briefly, 20,000 cells per well were seeded in 96-well plates and allowed to adhere overnight. The cells were then fixed with $4 \%$ paraformaldehyde, briefly permeabilized with Triton X-100, blocked, and incubated overnight at $4^{\circ} \mathrm{C}$ with primary antibodies. Primary antibodies were specific against mitochondrial DNA-encoded protein, COX-I, and nuclear DNA-encoded protein, SDH-A. The cells were washed and incubated for $1 \mathrm{~h}$ at room temperature with a solution of secondary antibodies containing an AP-labeled antibody specific for SDH-A and an HRP-labeled antibody specific for COX-I. The reactions were sequentially developed; first with an AP development solution, and then an HRP development solution. Fifteen minute kinetic reactions with 1 min intervals were recorded using a PHERAstar microplate reader (BMG Labtech) to measure optical density at $405 \mathrm{~nm}$ for AP development and $600 \mathrm{~nm}$ for HRP development. Whole cell staining with Janus Green was also done to compensate for variations due to cell loss during the procedure. The AP and HRP data were normalized to the Janus Green optical density at $595 \mathrm{~nm}$. The signal ratio of COX-I/SDH-A was calculated to determine increased production of mitochondrial DNA-encoded protein without a matching increase in nuclear DNA-encoded protein. Such an increase was interpreted as an increase in mitochondrial biogenesis. Statistical significance was measured using one-way ANOVA and Bonferroni's multiple comparisons test.

Statistical analysis. Each experiment was repeated at least three times. Data is presented as either mean values \pm standard deviation (SD), or as a percentage of the control. Statistical analysis was carried out using ordinary one- or two-way ANOVA with Bonferroni's multiple comparisons test where indicated. P-values $\leq 0.05$ were considered statistically significant.

\section{Results}

CoQ10 protects against cellular damage caused by glutamate exposure. Because the toxic effect of glutamate exposure can vary between cell lines, we first looked at glutamate-induced loss of viability in HT22 cells using a range of glutamate concentrations from 1 to $8 \mathrm{mM}$. The cells were exposed to these concentrations for $24 \mathrm{~h}$ before adding the resazurin dye as described previously (21). We observed a dose-dependent decrease in cell viability as glutamate concentration rises. At $4 \mathrm{mM}$, glutamate decreased viability by roughly $30 \%$ $(\mathrm{P}<0.001)$ and at the highest concentration of $8 \mathrm{mM},>80 \%$ of cells were died (data not shown). As a result, $4 \mathrm{mM}$ glutamate was selected for subsequent study. CoQ10 $(25 \mu \mathrm{g} / \mu \mathrm{l})$ treatment alone did not affect cell viability. However, CoQ10 pretreatment to glutamate-exposed cells effectively prevented the decline of cell viability caused by glutamate (data not shown).

In addition to reducing cell viability directly, cells surviving glutamate exposure may lose their proliferative capability even after glutamate has been removed. We assessed the the reproductive potential of HT22 cells after exposure to glutamate and/or CoQ10 treatment using clonogenic colony forming assay, an indication that they have retained their reproductive ability and are still capable of cellular division. Our experiments assessed this ability in cells that survived the initial $24 \mathrm{~h}$ glutamate exposure, CoQ10 treatment alone, or glutamate plus CoQ10 pretreatment. Surviving cells were harvested, washed and then seeded at low densities to allow individual cells to form visible colonies.

After staining colonies with crystal violet, the plating efficiency (PE) for each group was calculated by taking the number of colonies formed and dividing it by the number of cells seeded and then multiplying by 100 to obtain a percent. We found that in untreated cells, the average PE was 68\%. Our results showed the PE was reduced in glutamate-exposed cells dropping to $13 \%$, but was not significantly altered from control in the CoQ10 alone, or the glutamate plus CoQ10 group. To find the surviving fraction, we divided the PE of the treated sample by the PE of the control cells and multiplied by 100 . Value in untreated control group was set at $100 \%$ survival and our experimental groups were reported relative to this value. Glutamate exposure dropped survival to $19 \%$ ( $\mathrm{P}<0.001)$. CoQ10 alone and CoQ10 plus glutamate groups were not significantly different from untreated cells with average survival fractions of 93 and 101\% respectively (Fig. 1). In another word, CoQ10 reinstalled the cell survival.

CoQ10 promotes mitochondrial biogenesis. Labeling both a mitochondrial DNA-encoded protein (COX-I) and a nuclear DNA-encoded protein (SDH-A), we measured increases, or decreases, in COX-I expression over SDH-A that were interpreted as corresponding to increases, and decreases, in mitochondrial biogenesis, respectively. AP and HRP enzyme-catalyzed reactions (as described in methods) were developed for $15 \mathrm{~min}$ with optical density measured at 1 min intervals. Fig. 2A shows the ratio of the COX-I signal to the SDH-A signal (normalized to Janus Green) at each interval reading. The bar graph (Fig. 2B) 


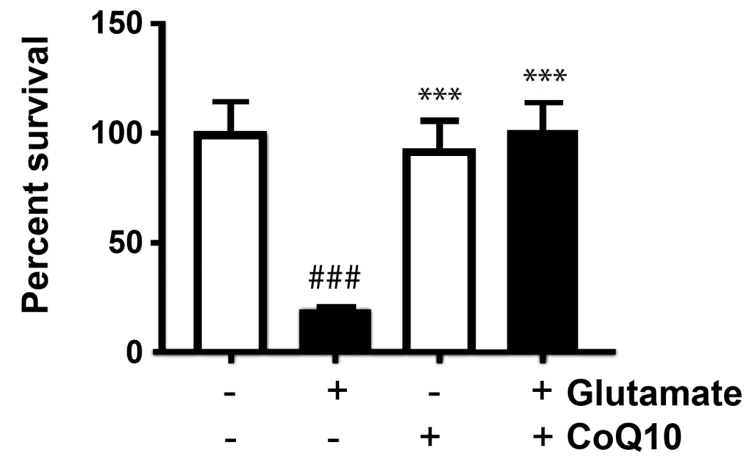

Figure 1. CoQ10 prevents the reduced cell survival induced by glutamate. The surviving fraction of cells was calculated as follows: PE of the treated sample/PE of the control cells $\times 100 .{ }^{\# \#} \mathrm{P}<0.001$ vs. control; ${ }^{* * *} \mathrm{P}<0.001$ vs. glutamate. CoQ10, coenzyme $\mathrm{Q} 10 ; \mathrm{PE}$, plating efficiency.

shows the average ratio for each experimental group at the 15 min endpoint. We observed that the COX-I/SDH-A ratio from glutamate exposure was moderately depressed indicating reduced mitochondrial biogenesis. CoQ10 treatment alone significantly increased mitochondrial biogenesis by roughly $60 \%$ compared to control $(\mathrm{P}<0.01)$. Combination of CoQ10 plus glutamate significantly increased the mitochondrial biogenesis compared to glutamate exposure alone $(\mathrm{P}<0.05)$.

CoQ10 reseved glutamate-suppressed $P G C-1 \alpha, p$-Akt and $p$-CREB. PGC- $1 \alpha$ is considered the master regulator of mitochondrial biogenesis through its action as a transcription factor for genes central in promoting mitochondrial growth. We show in Fig. 3 that glutamate exposure significantly reduced PGC- $1 \alpha$ expression by $40 \%$ in HT22 cells after $24 \mathrm{~h}(\mathrm{P}<0.01)$. Treatment with CoQ10 alone had no effect on PGC-1 $\alpha$ expression, but when added prior to glutamate addition, it significantly improved PGC-1 $\alpha$ expression, increasing it by $35 \%$ from glutamate exposure alone $(\mathrm{P}<0.05)$.

PGC-1 $\alpha$ is most commonly activated by either phosphorylation, or deacetylation, of its promoter region. A number of endogenous and exogenous actors can facilitate these reactions. One of noted inducers of PGC-1 $\alpha$ phosphorylation is the AKT/CREB pathway (20). Therefore, we measured expression of total and phosphorylated AKT and CREB in our experimental groups. The results showed that both phosphorylated AKT (p-AKT) and phosphorylated CREB (p-CREB) were moderately reduced from glutamate exposure albeit did not reach statistical significant. They were both upregulated when CoQ10 was added (Fig. 4; $\mathrm{P}<0.01$ for $\mathrm{p}$-CREB).

CoQ10 prevented glutamate-caused suppressions of NRF 2 and TFAM. The downstream effectors, NRF2 and TFAM, were also measured (Fig. 5). We observed a nearly $70 \%$ decrease in NRF2 expression in the nuclear fraction after glutamate exposure compared to the control $(\mathrm{P}<0.001)$. Addition of CoQ10 resulted in a complete restoration of NRF2 from this glutamate-inhibited level ( $\mathrm{P}<0.001$ vs. Glutamate). TFAM protein level was reduced by glutamate exposure. However, due to relative large variation, this reduction did not reach statistical significance. CoQ10 pretreatment to glutamate exposed cells resulted in a slight, but significantly increase in TFAM expression (20\%) compared to control $(\mathrm{P}<0.05)$.
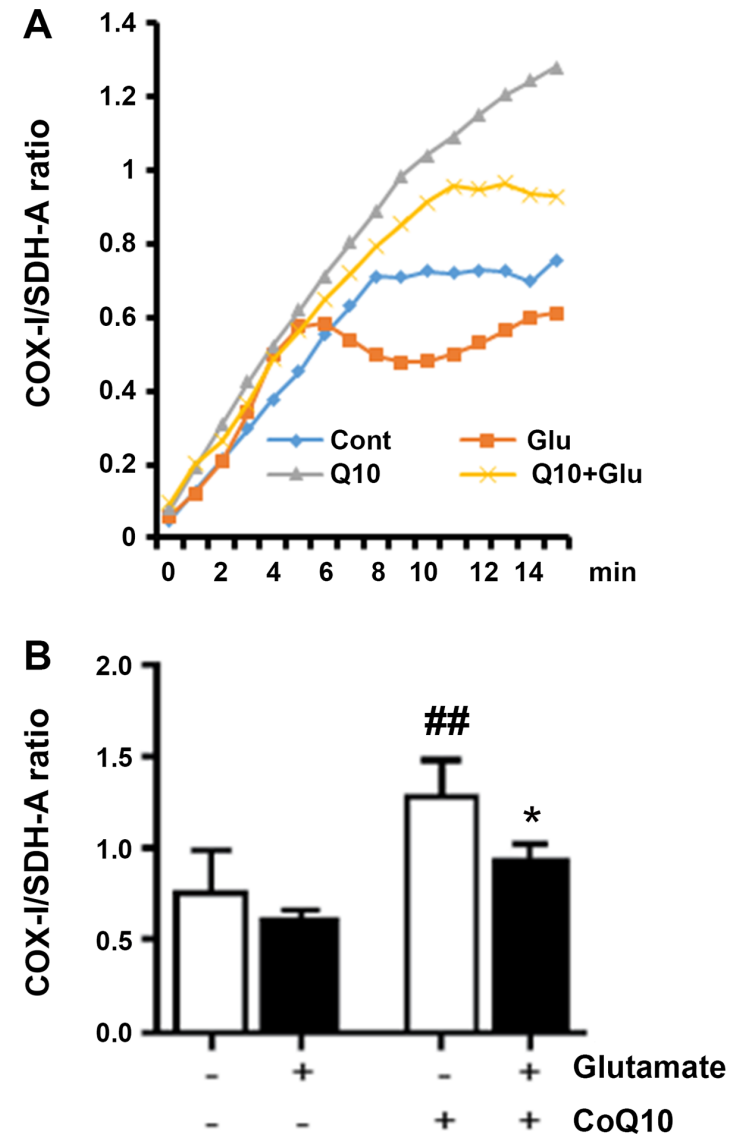

Figure 2. CoQ10 causes an increase in mitochondrial biogenesis. (A) ELISA-based mitochondrial biogenesis detection after treating cells with glutamate $(4 \mathrm{mM})$, CoQ10 $(25 \mathrm{mg} / \mathrm{l})$ or each in combination. COX-I optical density was detected via horseradish peroxidase enzyme development and SDH-A was detected via AP enzyme development for $15 \mathrm{~min}$. The graphed ratios are presented from the recorded optical densities of each reaction at $1 \mathrm{~min}$ intervals. The average results of at least three wells were taken. (B) Average ratio of mitochondrial DNA-encoded COX-I over nuclear DNA-encoded SDH-A at the 15 min development endpoint of each treatment group. ${ }^{\# \#} \mathrm{P}<0.01$ vs. control and ${ }^{*} \mathrm{P}<0.05$ vs. glutamate. CoQ10, coenzyme Q10; COX-I, cyclooxygenase I; SDH-A, uccinate dehydrogenase complex flavoprotein subunit A; AP, alkaline phosphate.

\section{Discussion}

The present study showed that glutamate significantly reduced cell viability and reproductivity (cell proliferation) after glutamate exposure. Pretreatment with CoQ10 $24 \mathrm{~h}$ prior to glutamate exposure significant limited the damaging effects of glutamate on cell viability and cell proliferation capacity after glutamate exposure. These results are consistent with our previous published results $(4,5,24,25)$. The glutamate toxicity is time-dependent, with mild damage observed after $10 \mathrm{~h}$, decreased to about $40-60 \%$ after $24 \mathrm{~h}$, and further reduced to less than $20 \%$ of the control value after 36 and $48 \mathrm{~h}(5,25)$. It is likely that glutamate caused reduction in cell viability is caused by cell death. We have previously shown that glutamate exposure increases ROS production, causes mitochondrial membrane hyperpolarization, suppressed mitochondrial respiration and resulted in mitochondrial fragmentation. Furthermore, glutamate increases the release of AIF to nuclei, activates caspases 9 and 3, and resulted in TUNEL positive cell death $(4,5,24,25)$. 
A

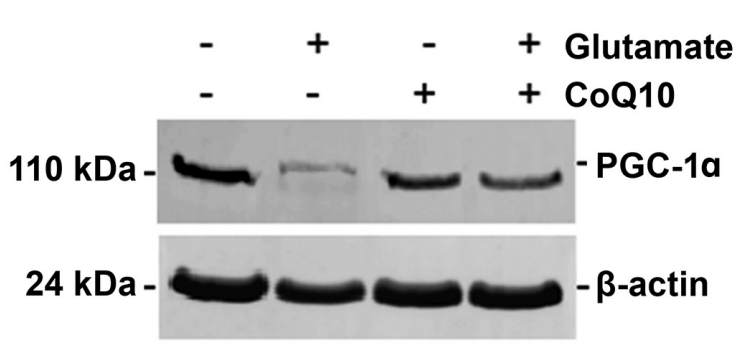

B

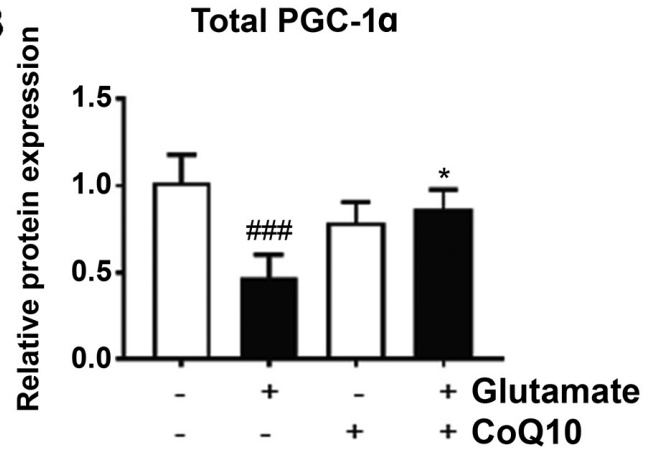

Figure 3. CoQ10 pretreatment prevents the reduction of PGC-1 $\alpha$ protein expression induced by glutamate. (A) Representative western blotting images for

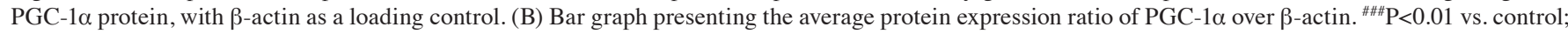
${ }^{*} \mathrm{P}<0.05$ vs. glutamate. Results are representative of at least three western blots. CoQ10, coenzyme Q10; PGC-1 $\alpha$, peroxisome proliferator-activated receptor gamma coactivator- $1 \alpha$.
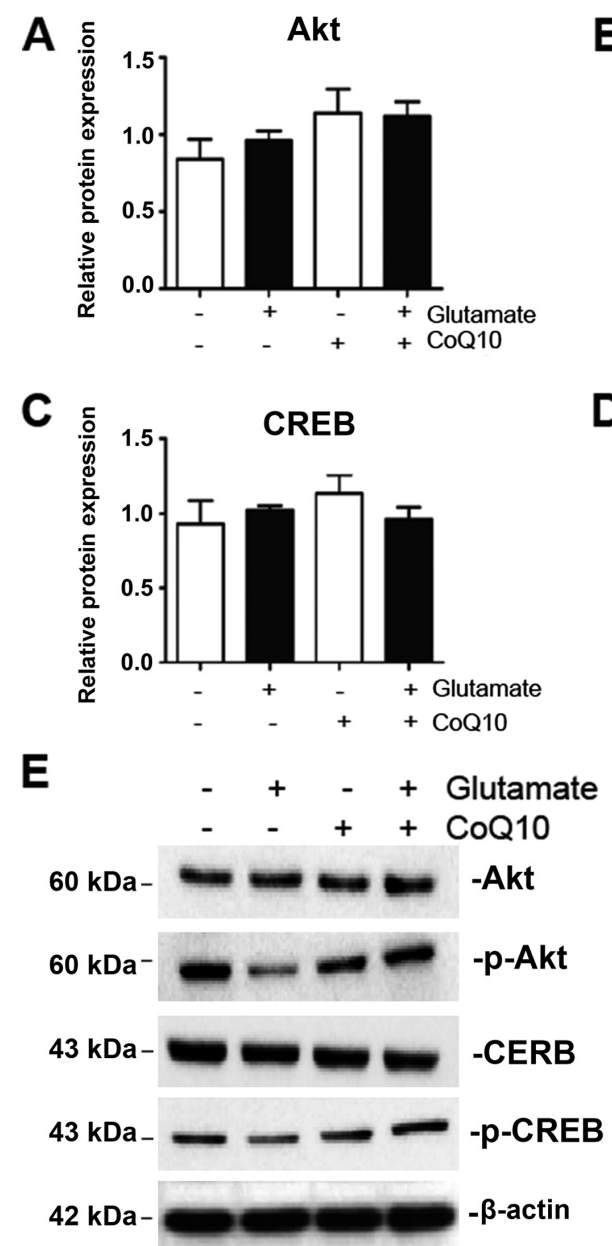
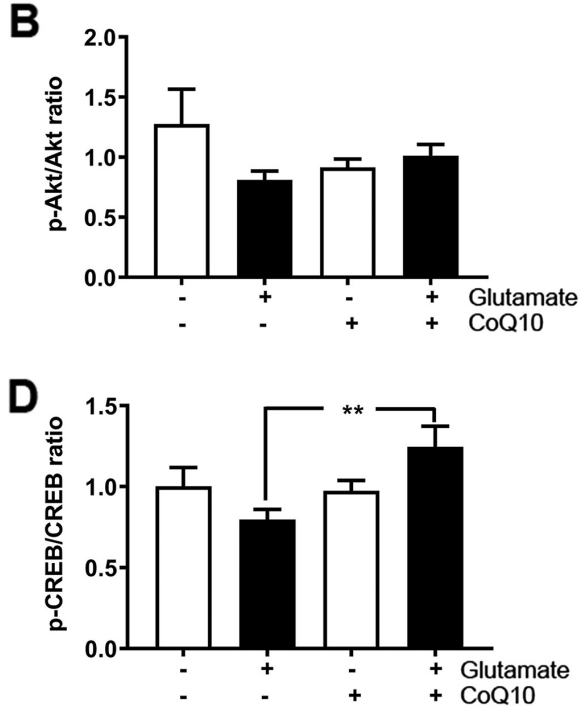

Figure 4. CoQ10 reversed the Glutamate-induced suppressions of p-Akt and p-CREB. (A) Relative protein band density of Akt. (B) Relative protein band density of p-Akt. (C) Relative protein band density of CREB. (D) Relative protein band density of p-CREB over $\beta$-actin. (E) Representative western blots using nuclear fraction. Results are representative of at least three western blots. ${ }^{* *} \mathrm{P}<0.01$ vs. Glutamate. CoQ10, coenzyme Q10; p, phosphorylated; CREB, cAMP response element-binding protein.

Damage from glutamate excitotoxicity is exacerbated by oxidative stress (5) and increasing the number of mitochondria is a way to boost the antioxidant, detoxification functions of cells against this stress. A study by Wang et al, showed that $\mathrm{NAD}^{+}$protected against glutamate-induced apoptosis through increasing mitochondrial biogenesis (26). We believe CoQ10 similarly protects against cell death by boosting mitochondrial biogenesis. Protecting PGC-1 $\alpha$ expression may be crucial to this effect. Our results demonstrated that glutamate markedly suppressed PGC-1 $\alpha$ protein level and CoQ10 treatment provided restoration of glutamate-diminished PGC-1 $\alpha$ protein expression, and at the same time increased cell viability and proliferative potential. It is interesting that CoQ10 treatment alone significantly increased mitochondrial biogenesis without 
A

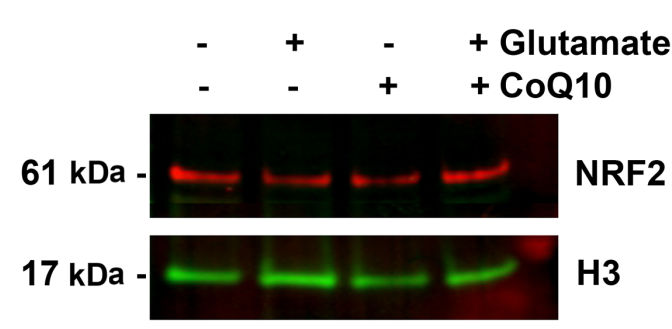

B

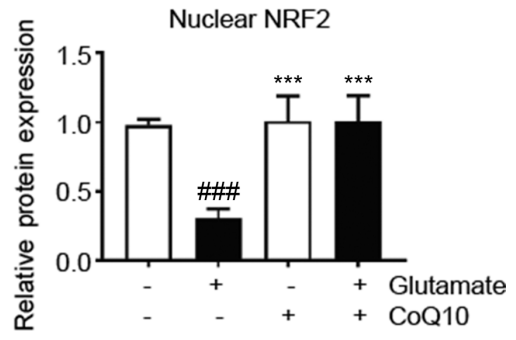

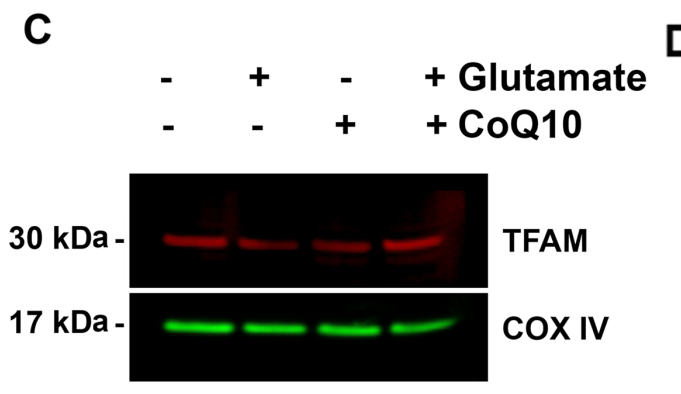

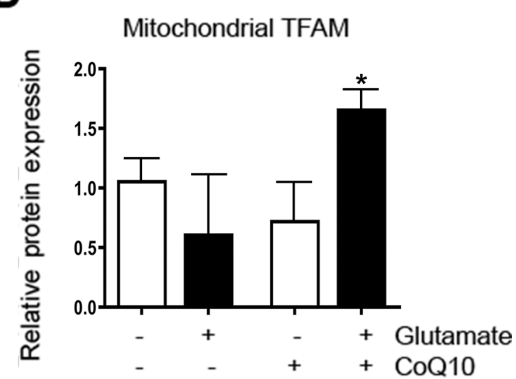

Figure 5. CoQ10 increased NRF2 and TFAM. (A) Representative western blotting images of NRF2 in the nuclear fraction. The results demonstrated that glutamate reduced the protein expression of NRF2, which was restored by CoQ10. (B) Average nuclear protein expression of NRF2 relative to an untreated control. Protein content was normalized to H3. (C) Representative western blotting images of TFAM in the mitochondrial fraction. CoQ10 treatment increased the expression of TFAM. (D) Average mitochondrial protein expression of TFAM relative to an untreated control. Protein content was normalized to COX-IV. Results from at least three separate experiments are presented. ${ }^{*} \mathrm{P}<0.05$ and ${ }^{* * * *} \mathrm{P}<0.001$ vs. control; ${ }^{\# \#} \mathrm{P}<0.001 \mathrm{vs}$. glutamate. CoQ10, coenzyme $\mathrm{Q} 10$; NRF, nuclear respiratory factor; TFAM, mitochondrial transcription factor A; H3, histone H3; COX-VI, cyclooxygenase VI.

much increase in PGC-1 $\alpha$. The reason for this phenomenon is not quite clear but could be because there were other unknown factors except PGC-1a regulating mitochondrial biogenesis, PGC-1 $\alpha$ was upregulated at transcription level, or a mild upregulation of PGC-1 $\alpha$ was sufficient to increase the synthesis of mitochondrial DNA-encoded proteins. We expanded our understanding of this mechanism by exploring alterations to PGC-1 $\alpha$ signaling pathways both upstream and downstream this central regulator.

Among the many upstream facts that regulate PGC- $1 \alpha$, we measured AKT and CREB. While the suppressive effect of glutamate on p-Akt and pCREB was moderate, treatment with CoQ10 in combination of glutamate increased p-Akt and p-CREB levels, suggesting upregulation of PGC- $1 \alpha$ and mitochondrial biogenesis by CoQ10 may involve phosphorylation of Akt and CREB.

NRF1,2 and TFAM are the known downstream targets of PGC- $1 \alpha$. While we did not observe a change in NRF1 in our model, we did observe significant degradation of NRF2 from glutamate exposure. NRF2 is a transcription factor, activated under stress conditions, to increase survival through enhancing transcription of genes involved in anti-inflammatory, antioxidant, and general cytoprotective pathways (27). Stimulated by PGC-1 $\alpha$, NRF2 has been shown to be a key contributor to mitochondrial biogenesis pathways $(28,29)$. NRF2's upregulation after CoQ10 treatment may thus contribute to our observed increase in mitochondrial biogenesis. This effect could be achieved either directly activating mitochondria DNA and protein synthesis or indirectly through activating TFAM $(30,31)$. TFAM is an important mitochondrial transcription factor integral to maintaining the mitochondrial genome by regulating its packaging, stability, and replication (32). While we did not observe a significant decrease in TFAM after glutamate exposure, it was significantly elevated by CoQ10 treatment. This suggest that TFAM may or may not involve glutamate-caused suppression in mitochondrial biogenesis signaling, but it participated surely in CoQ10 stimulated mitochondrial biogenesis.

In summary, our results show that glutamate, while causing severe cellular damage, affects mitochondrial biogenesis pathways, particularly through inhibition of PGC-1 $\alpha$, to decrease mitochondrial biogenesis. CoQ10 is an effective option for mitigating this damage. In addition to preventing decreases in PGC- $1 \alpha$, CoQ10 stimulates p-AKT, p-CREB, NRF2, and TFAM. Glutamate toxicity is involved in the pathogenesis of many neurodegenerative disorders and CoQ10 blocks the glutamate toxicity through stimulating mitochondrial biogenesis. Therefore, CoQ10 could be considered as complementary treatment for neurological diseases. Further exploration of mechanisms of CoQ10-mediated mitochondrial biogenesis may improve and broaden the clinical application of CoQ10.

\section{Acknowledgements}

Not applicable.

\section{Funding}

The current study was supported by the National Natural Science Foundation of China (grant no. 8156050251 to GC). 


\section{Availability of data and materials}

The datasets used and/or analyzed during the current study are available from the corresponding author on reasonable request.

\section{Authors' contributions}

GC and PAL conceived and designed the experiments of the current study. MAZ, MH, QQ and SLM performed the experiments. MAZ, GC, PAL wrote the manuscript. All authors read and approved the final manuscript.

\section{Ethics approval and consent to participate}

Not applicable.

\section{Patient consent for publication}

Not applicable.

\section{Competing interests}

The authors declare that they have no competing interests.

\section{References}

1. Olivares-Banuelos TN, Chí-Castañeda D and Ortega A: Glutamate transporters: Gene expression regulation and signaling properties. Neuropharmacology 161: 107550, 2019.

2. Amantea D and Bagetta G: Excitatory and inhibitory amino acid neurotransmitters in stroke: From neurotoxicity to ischemic tolerance. Curr Opin Pharmacol 35: 111-119, 2017.

3. You J, Feng L, Xin M, Ma D and Feng J: Cerebral ischemic postconditioning plays a neuroprotective role through regulation of central and peripheral glutamate. Biomed Res Int 2018: 6316059, 2018.

4. Kumari S, Mehta SL, Milledge GZ, Huang X, Li H and Li PA: Ubisol-Q10 prevents glutamate-induced cell death by blocking mitochondrial fragmentation and permeability transition pore opening. Int J Biol Sci 12: 688-700, 2016.

5. Kumari S, Mehta SL and Li PA: Glutamate induces mitochondria dynamic imbalance and autophagy activation: Preventive effects of selenium. PLoS One 7: e39382, 2012.

6. Jing L, Kumari S, Mendelev N and Li PA: Coenzyme Q10 ameliorates ultraviolet $\mathrm{B}$ irradiation induced cell death through inhibition of mitochondrial intrinsic cell death pathway. Int J Mol Sci 12: 8302-8315, 2011

7. Li H, Chen G, Ma W and Li PA: Water-soluble coenzyme Q10 inhibits nuclear translocation of apoptosis inducing factor and cell death caused by mitochondrial complex I inhibition. Int J Mol Sci 15: 13388-13340, 2014.

8. Jing L, He MT, Chang Y, Mehta SL, He QP, Zhang JZ and Li PA Coenzyme Q10 protects astrocytes from ROS-induced damage through inhibition of mitochondrial-mediated cell death pathways. Int J Biol Sci 11: 59-66, 2015.

9. Li HN, Zimmerman M, Milledge GZ, Hou XL, Cheng J, Wang ZH and Li PA: Water soluble coenzyme Q10 reduced rotenone-induced mitochondrial fission. Neurochem Res 42: 1096-1103, 2017.

10. Niyazov DM, Kahler SG and Frye RE: Primary mitochondrial disease and secondary mitochondrial dysfunction: Importance of distinction for diagnosis and treatment. Mol Syndromol 7: 122-137, 2016.

11. Failla ML, Chitchumroonchokchai $\mathrm{C}$ and Aoki F: Increased bioavailability of ubiquinol compared to that of ubiquinone is due to more efficient micellarization during digestion and greater GSH-dependent uptake and basolateral secretion by Caco-2 cells. J Agric Food Chem 62: 7174-7182, 2014.

12. Kalen A, Appelkvist EL and Dallner G: Age-related changes in the lipid compositions of rat and human tissues. Lipids 24: 579-584, 1989.
13. Mortenson SA, Rosenfeldt F, Kumar A, Dolliner P, Filipiak KJ Pella D, Alehagen U, Steurer G and Littarru GP: Q-SYMBIO study investigators: The effect of coenzyme Q10 on morbidity and mortality in chronic heart failure: Results from Q-SYMBIO: A randomized double-blind trial. JACC Heart Fail 2: 641-649, 2014.

14. Folkers K and Simonsen R: Two successful double-blind trials with coenzyme Q10 (vitamin Q10) on muscular dystrophies and neurogenic atrophies. Biochim Biophys Acta 1271: 281-286, 1995.

15. Fallah M Askari G, Soleimani A, Feizi A and Asemi Z: Clinical trial of the effects of coenzyme Q10 supplementation on biomarkers of inflammation and oxidative stress in diabetic hemodialysis patients. Int J Prev Med 10: 12, 2019.

16. Sanoobar M, Dehghan P, Khalili M, Azimi A and Seifar F: Coenzyme Q10 as a treatment for fatigue and depression in multiple sclerosis patients: A double blind randomized clinical trial. Nutr Neurosci 19: 138-143, 2016.

17. Valero T: Mitochondrial biogenesis: Pharmacological approaches. Curr Pharm Des 20: 5507-5509, 2014.

18. Uittenbogaard $\mathrm{M}$ and Chiaramello A: Mitochondrial biogenesis: A therapeutic target for neurodevelopmental disorders and neurodegenerative diseases. Curr Pharm Des 20: 5574-5593, 2014.

19. Procaccio V, Bris C, Chao de la Barca JM, Oca F, Chevrollier A, Amati-Bonneau P, Bonneau D and Reynier P: Perspectives of drug-based neuroprotection targeting mitochondria. Rev Neurol (Paris) 170: 390-400, 2014

20. Li PA, Hou X and Hao S: Mitochondrial biogenesis in neurodegeneration. J Neurosci Res 95: 2025-2029, 2017.

21. Zimmerman MA, Biggers CD and Li PA: Rapamycin treatment increases hippocampal cell viability in an mTOR-independent manner during exposure to hypoxia mimetic, cobalt chloride. BMC Neurosci 19: 82, 2018.

22. Guzman C, Bagga M, Kaur A, Westermarck J and Abankwa D: ColonyArea: an ImageJ plugin to automatically quantify colony formation in clonogenic assays. PLoS ONE 9:e92444, 2014.

23. Andrews NC and Faller DV: A rapid micro-preparation technique for extraction of DNA-binding proteins from limiting numbers of mammalian cells. Nucleic Acids Res 19: 2499, 1991.

24. Mehta SL, Kumari S, Mendelev N and Li PA: Selenium preserves mitochondrial function, stimulates mitochondrial biogenesis, and reduces infarct volume after focal cerebral ischemia. BMC Neurosci 13: 79, 2012

25. Ma YM, Ibeanu G, Wang LY, Zhang JZ, Chang C, Dong JD, Li PA and Jing L: Selenium suppresses glutamate-induced cell death and prevents mitochondrial morphological dynamic alterations in hippocampal HT22 neuronal cells. BMC Neurosci 18: $15,2017$.

26. Wang $\mathrm{X}, \mathrm{Li} \mathrm{H}$ and Ding $\mathrm{S}$ : The effects of NAD+ on apoptotic neuronal death and mitochondrial biogenesis and function after glutamate excitotoxicity. Int J Mol Sci 15: 20449-20468, 2014.

27. Dinkova-Kostova AT and Abramov AY: The emerging role of Nrf2 in mitochondrial function. Free Radic Biol Med 88: 179-188, 2015.

28. Hayashi G, Jasoliya M, Sahdeo S, Saccà F, Pane C, Filla A, Marsili A, Puorro G, Lanzillo R, Brescia Morra V and Cortopassi G: Dimethyl fumarate mediates Nrf2-dependent mitochondrial biogenesis in mice and humans. Hum Mol Genet 26: 2864-2873, 2017.

29. Bernard K, Logsdon NJ, Miguel V, Benavides GA, Zhang J, Carter AB, Darley-Usmar VM and Thannickal VJ: NADPH oxidase 4 (Nox4) suppresses mitochondrial biogenesis and bioenergetics in lung fibroblasts via a nuclear factor erythroid-derived 2-like 2 (Nrf2)-dependent pathway. J Biol Chem 292: 3029-3038, 2017.

30. Chaturvedi RK and Beal MF: Mitochondrial diseases of the brain. Free Radic Biol Med 63: 1-29, 2013

31. Khatua TN, Dinda AK, Putcha UK and Banerjee SK: Diallyl disulfide ameliorates isoproterenol induced cardiac hypertrophy acting mitochondrial biogenesis via eNOS-Nrf2-Tfam pathway in rats. Biochem Biophys Rep 5: 77-88, 2015.

32. Kang I, Chu CT and Kaufman BA: The mitochondrial transcription factor TFAM in neurodegeneration: Emerging evidence and mechanisms. FEBS Lett 592: 793-811, 2018.

This work is licensed under a Creative Commons

Attribution-NonCommercial-NoDerivatives 4.0 International (CC BY-NC-ND 4.0) License. 